\title{
THE NATURAL TOPOLOGY OF MATLIS REFLEXIVE MODULES
}

\author{
RICHARD BELSHOFF
}

\begin{abstract}
For a complete local ring $R$ with maximal ideal $m$, we define a linear topology on a Matlis reflexive $R$-module $M$ which coincides with the $m$-adic topology on $M$ in case $M$ is finitely generated. We show that a Matlis reflexive module is complete in this topology.
\end{abstract}

\section{INTRODUCTION}

Let $(R, m)$ be a local noetherian ring and let $E$ denote the injective envelope of $R / \mathrm{m}$. For an $R$-module $M$ the Matlis dual $\operatorname{Hom}_{R}(M, E)$ is denoted $M^{\nu}$, and $M$ is said to be Matlis reflexive if the canonical injection $M \rightarrow M^{\nu \nu}$ is an isomorphism. It is well known [6, Theorem 3.7, p.522] that there is a canonical isomorphism $R^{\nu \nu} \cong \widehat{R}$, where $\widehat{R}$ denotes the $\mathrm{m}$-adic completion of $R$, and so any complete local ring $R$ is a Matlis reflexive $R$-module. It follows that $M^{\nu \nu} \cong \widehat{M}$ if $M$ is a finitely generated $R$-module, because in this case, $M^{\nu \nu} \cong M \otimes_{R} \operatorname{Hom}_{R}(E, E) \cong M \otimes_{R} \widehat{R} \cong \widehat{M}$.

For the rest of this article $R$ will denote a complete local ring. In this case, finitely generated and artinian $R$-modules are Matlis reflexive. Also, $M$ is an artinian (noetherian) $R$-module if and only if $M^{\nu}$ is noetherian (artinian). This is the essence of what is commonly known as "Matlis duality". See [6, Corollary 4.3, p.528] for more details. The main results of Matlis [6] are nicely presented in Matsumura's recent book [7].

Enochs [5, Proposition 1.3, p.181] has shown that an $R$-module $M$ is Matlis reflexive if and only if $M$ has a finitely generated submodule $S$ such that $M / S$ is artinian. This characterisation has proved to be very useful. For example, in [2] it was used to show that if $M$ and $N$ are Matlis reflexive $R$-modules, then so are $\operatorname{Hom}_{R}(M, N)$, $M \otimes_{R} N$, and more generally $\operatorname{Ext}_{R}^{n}(M, N)$ and $\operatorname{Tor}_{n}^{R}(M, N)$ for all $n \geqslant 1$.

In section 2 we use this characterisation to define a linear topology on a Matlis reflexive $R$-module $M$, which we call the Matlis topology, or the natural topology of the Matlis reflexive $R$-module $M$. This topology is a generalisation of the usual $\mathrm{m}$-adic topology in the sense that these two topologies coincide when $M$ is finitely generated. For general references on linear topologies and completions, see $[1,3$, or 7$]$. In Section

Received 10th February 1992.

It is a pleasure to acknowledge the help given to me by Professor Edgar Enochs.

Copyright Clearance Centre, Inc. Serial-fee code: 0004-9729/93 \$A2.00+0.00. 
3 we show that for a finitely generated submodule $S$ of a Matlis reflexive $R$-module $M$, the subspace topology that $S$ inherits from $M$ is the same as the m-adic topology on $S$. In Section 4 we show that a Matlis reflexive module is complete in its natural topology.

\section{Definition of the Natural Topology}

In this section we show that a Matlis reflexive module $M$ has a natural topology defined in terms of the finitely generated submodules $S \subset M$ such that $M / S$ is artinian. If we choose a particular finitely generated submodule $S \subset M$ with $M / S$ artinian, then we have $S \supset \mathrm{m} S \supset \mathrm{m}^{2} S \supset \cdots$, a decreasing filtration on $M$. And so there is a topology on $M$ such that $\left\{\mathrm{m}^{n} S\right\}$ form a fundamental system of neighbourhoods of 0 , called the topology on $M$ defined by the filtration $\left\{\mathrm{m}^{n} S\right\}$. Let $M_{n}=\mathrm{m}^{n} S$ for $n \geqslant 0$, and $M_{n}=M$ for $n<0$. Then the filtration $\left(M_{n}\right)_{n \in Z}$ is such that $\bigcup M_{n}=M$, and $\cap M_{n}=0$ and so is exhaustive and separated (Hausdorff.)

We begin with an easy Lemma.

LEMma 1.

1. If $S \subset M$ is a finitely generated submodule such that $M / S$ is artinian, then $\mathrm{m} S \subset M$ is also finitely generated with $M / \mathrm{m} S$ artinian.

2. If $S_{1}, S_{2} \subset M$ are two finitely generated submodules with $M / S_{1}, M / S_{2}$ artinian, then $S_{1} \cap S_{2} \subset M$ is also finitely generated and $M / S_{1} \cap S_{2}$ is artinian.

Proof: 1 . Clearly $\mathrm{m} S$ is finitely generated; and the kernel of the induced surjection $M / \mathrm{m} S \longrightarrow M / S \rightarrow 0$ is $S / \mathrm{m} S$, which is a finite dimensional vector space and so is artinian. Therefore $M / \mathrm{m} S$ is artinian.

For 2, it's clear that $S_{1} \cap S_{2}$ is finitely generated. And $M /\left(S_{1} \cap S_{2}\right)$ is isomorphic to a submodule of the artinian module $M / S_{1} \times M / S_{2}$, and so is artinian.

If we had chosen another finitely generated submodule $T \subset M$ with $M / T$ artinian to define the topology, then by part (3) of the next Lemma, both $S$ and $T$ give rise to the same topology on $M$. We will call this topology the natural topology of the Matlis reflexive module $M$. So a basic neighbourhood of 0 will be any finitely generated submodule $S \subset M$ such that $M / S$ is artinian.

Lemma 2. Suppose that an $R$-module $M$ has a finitely generated submodule $S \subset M$ such that $M / S$ is artinian. Let $T \subset M$ be any submodule. Then the following are equivalent:

(1) $T$ is finitely generated and $M / T$ is artinian.

(2) Both $(S+T) / S$ and $(S+T) / T$ have finite length.

(3) For some integer $n, \mathrm{~m}^{n} S \subset T$ and $\mathfrak{m}^{n} T \subset S$. 
Proof: (1) $\Rightarrow(2)$. Since $T$ is finitely generated, the image $T /(S \cap T)$ is too. But $T /(S \cap T) \cong(S+T) / S$ is also artinian, since it's a submodule of the artinian module $M / S$, and similarly for $(S+T) / T$.

(2) $\Longrightarrow(3)$. If $(S+T) / T$ has finite length, then since

$$
(S+T) / T \supset(\mathrm{m} S+T) / T \supset\left(\mathrm{m}^{2} S+T\right) / T \supset \cdots,
$$

we must have $\left(\mathrm{m}^{n} S+T\right) / T=0$ for some integer $n$. Hence $\mathrm{m}^{n} S+T=T$ for some $n$ and so we have $m^{n} S \subset T$. Similarly, if $(S+T) / S$ is of finite length, then use the descending chain

$$
(S+T) / S \supset(S+\mathrm{m} T) / S \supset\left(S+\mathrm{m}^{2} T\right) / S \supset \cdots
$$

to get that $\mathrm{m}^{n} T \subset S$.

(3) $\Longrightarrow$ (1). Since $m^{n} T \subset S$ and $S$ is finitely generated, then $m^{n} T$ is finitely generated. And $M / \mathrm{m}^{n} T$ is artinian. Then $T / \mathrm{m}^{n} T \subset M / \mathrm{m}^{n} T$ is also artinian. And $T / \mathrm{m}^{n} T$ is a module over $R / \mathrm{m}^{n}$, an artinian ring. So $T / \mathrm{m}^{n} T$ is finitely generated. Then it follows that $T$ is finitely generated. The induced onto homomorphism $M / \mathrm{m}^{n} S \longrightarrow$ $M / T \longrightarrow 0$ and the fact that $M / \mathrm{m}^{n} S$ is artinian show that $M / T$ is artinian.

The next result is a generalisation of "Chevalley's theorem". The theorem states that if $(R, \mathrm{~m})$ is a complete local ring, $M$ is a finitely generated $R$-module and $\left(S_{t}\right)_{t=1}^{\infty}$ is a nonincreasing sequence of submodules such that $\bigcap_{t=1}^{\infty} S_{t}=0$, then for every $n$ there exists $t(n)$ such that $S_{t(n)} \subset \mathrm{m}^{n} M$.

In other words given a finitely generated module over a complete local ring, if a nonincreasing sequence of submodules has intersection 0 then the terms are eventually contained in a large power of the maximal ideal times the module. (See $[4,7$, Exercise 8.7, p.63, or 8 ].)

We show next that Chevalley's theorem is true not only for finitely generated modules but also for Matlis reflexive modules. So Chevalley's theorem is valid in the special case when $M$ is artinian.

Proposition 1 . Let $M$ be an $R$-module with a finitely generated submodule $T$ such that $M / T$ is artinian. If

$$
S_{1} \supset S_{2} \supset \cdots \supset S_{n} \supset S_{n+1} \supset \cdots
$$

is any chain of submodules of $M$ such that $\bigcap_{n=1}^{\infty} S_{n}=0$, then for every $n$ there exists an integer $s(n)$ such that $S_{s(n)} \subset \mathfrak{m}^{n} T$.

PROOF: If the conclusion is not true, then there exists an integer $n_{0}$ so that for every $s, S, \not \subset \mathrm{m}^{n} T$ for all $n \geqslant n_{0}$. Since $M / \mathrm{m}^{n} T$ is artinian for every $n$, the chain

$$
M / m^{n} T \supset\left(S_{1}+m^{n} T\right) / m^{n} T \supset\left(S_{2}+m^{n} T\right) / m^{n} T \supset \cdots
$$


stabilises, so for each $n$ there exists an integer $s(n)$ such that

$$
S_{s(n)}+\mathfrak{m}^{n} T=S_{s(n)+1}+\mathfrak{m}^{n} T=S_{s(n)+2}+\mathfrak{m}^{n} T=\cdots
$$

Note that we can take $s(0)<s(1)<s(2)<\cdots$. Also note that since $S, \not \subset \mathrm{m}^{n} T$ for all $s$ and for all $n \geqslant n_{0}$, we have $S_{\bullet}+m^{n} T \neq m^{n} T$ for all $s$ and for all $n \geqslant n_{0}$.

Next we construct a sequence. There exists an element $x_{n_{0}} \in S_{\triangleleft\left(n_{0}\right)}$ with $x_{n_{0}} \notin$ $\mathrm{m}^{\mathrm{n}} \mathrm{T}$. Since

$$
S_{a\left(n_{0}\right)}+\mathrm{m}^{n_{0}} T=S_{\left(n_{0}\right)+1}+\mathrm{m}^{n_{0}} T=\cdots=S_{\iota\left(n_{0}+1\right)}+\mathrm{m}^{n_{0}} T=\cdots,
$$

there exists $x_{n_{0}+1} \in S_{\alpha\left(n_{0}+1\right)}$ such that $x_{n_{0}}=x_{n_{0}+1}+y_{n_{0}}$ for some $y_{n_{0}} \in \mathrm{m}^{n_{0}} T$. And since

$$
S_{a\left(n_{0}+1\right)}+m^{n_{0}+1} T=S_{s\left(n_{0}+1\right)+1}+m^{n_{0}+1} T=\cdots=S_{a\left(n_{0}+2\right)}+m^{n_{0}+1} T=\cdots,
$$

there exists $x_{n_{0}+2} \in S_{a\left(n_{0}+2\right)}$ such that $x_{n_{0}+1}=x_{n_{0}+2}+y_{n_{0}+1}$ for some $y_{n_{0}+1} \in$ $\mathrm{m}^{n_{0}+1} T$. Continuing in this way we get a sequence $x_{n_{0}}, x_{n_{0}+1}, x_{n_{0}+2}, \cdots$ of points of $T$ such that $x_{n_{0}+k}-x_{n_{0}+k+1} \in \mathrm{m}^{n_{0}+k} T$ for all integers $k \geqslant 0$ and so this sequence is a Cauchy sequence.

Since $T$ is finitely generated over a complete local ring $R$, then $T$ is complete and so there exists an element $x=\lim _{i \rightarrow \infty} x_{n_{0}+i}$. Note that $x \in S_{\lrcorner\left(n_{0}+k\right)}$ for all $k \geqslant 0$, and so $x \in \bigcap_{i=0}^{\infty} S_{a\left(n_{0}+i\right)}$. Therefore $x=0$.

And by definition of limit, there exists an $N$ so that for $i \geqslant N$ we have $x_{n_{0}+i} \in$ $m^{n_{0}} T$. But we have

$$
x_{n_{0}}+m^{n_{0}} T=x_{n_{0}+1}+m^{n_{0}} T=x_{n_{0}+2}+m^{n_{0}} T=\cdots=x_{n_{0}+N}+m^{n_{0}} T=\cdots=
$$

and so, since $x_{n_{0}+N} \in \mathrm{m}^{n_{0}} T$ we see that $x_{n_{0}} \in \mathrm{m}^{n_{0}} T$, a contradiction.

\section{Comparison of Topologies}

Given a Matlis reflexive module $M$, choose a finitely generated submodule $T \subset M$ with $M / T$ artinian so that $\left\{\mathrm{m}^{n} T\right\}$ is a fundamental system of neighbourhoods of 0 . Let $S \subset M$ be any finitely generated submodule. Then $S$ has two topologies, $\mathcal{T}$ and $\mathcal{T}^{\prime}$, both given by systems of neighbourhoods of 0 . One is the neighbourhood base $\left\{\mathrm{m}^{\mathrm{n}} S\right\}$ of 0 giving us the topology $\mathcal{T}$, the usual m-adic topology on $S$. Another is the induced neighbourhood base $\left\{\mathrm{m}^{n} T \cap S\right\}$ giving an induced topology $\mathcal{T}^{\prime}$, when $S$ is thought of as a subspace of $M$ with its natural topology. We first show that these two topologies on $S, \mathcal{T}$ and $\mathcal{T}^{\prime}$, are the same. 
By Proposition 1, given any $n$ there exists an integer $s(n)$ so that $m^{\circ(n)} S \subset m^{n} T$. Therefore $m^{\circ(n)} S \subset m^{n} T \cap S$. This shows that any set which is open in $\mathcal{T}^{\prime}$, the induced Matlis topology on $S$, is also open in $\mathcal{T}$, the m-adic topology on $S$. That is $\mathcal{T}^{\prime} \subset \mathcal{T}$, or $\mathcal{T}$ is finer than $\mathcal{T}^{\prime}$.

To show that $\mathcal{T}^{\prime}$ is finer than $\mathcal{T}$ it would be enough to prove the Artin-Rees Lemma, that is, for some $n_{0}$,

$$
m^{n}\left(m^{n_{0}} T \cap S\right)=m^{n_{0}+n} T \cap S
$$

for all $n$. Since given any neighbourhood $\mathrm{m}^{k} S$, we certainly have

$$
\mathrm{m}^{k}\left(\mathrm{~m}^{n_{0}} T \cap S\right) \subset \mathrm{m}^{k} S
$$

but then by (1) we would get

$$
\mathrm{m}^{n_{0}+k} T \cap S \subset \mathrm{m}^{k} S
$$

We now prove (1). Since $R$ is noetherian, the graded ring $R^{*}=\oplus_{n=0}^{\infty} \mathrm{m}^{n}$ is also noetherian. And the graded module $T^{*}=\oplus_{n=0}^{\infty} \mathrm{m}^{n} T$ is a finitely generated $R^{*}$-module. Now we look at the filtration on $S$,

$$
S \supset T \cap S \supset \mathrm{m} T \cap S \supset \mathrm{m}^{2} T \cap S \supset \cdots
$$

where we set $S_{n}:=\mathrm{m}^{n} T \cap S$ for $n \geqslant 0$. Then $\mathrm{m} S_{n} \subset S_{n+1}$, and so $S^{*}:=\oplus_{n=0}^{\infty} S_{n}$ is an $R^{*}$-module. In fact, $S^{*}$ is an $R^{*}$-submodule of $T^{*}$, and so is a finitely generated $R^{*}$-module. Hence $\left[1\right.$, Lemma 10.8, p.107] for some $n_{0}$ we have $\mathrm{m} S_{n}=S_{n+1}$ for all $n \geqslant n_{0}$. So we have

$$
\mathrm{m}\left(\mathrm{m}^{n_{0}} T \cap S\right)=\mathrm{m}^{n_{0}+1} T \cap S
$$

which proves (1). So the two topologies $\mathcal{T}$ and $\mathcal{T}^{\prime}$ on the finitely generated submodule $S$ are the same.

\section{A Reflexive Module is Complete}

Suppose now that $\left(x_{n}\right)$ is a Cauchy sequence in $M$, a Matlis reflexive $R$-module, where $M$ is given its natural Matlis topology. This means that given any basic neighbourhood $S$ of 0 , there exists an $n_{0}$ so that $x_{n}-x_{m} \in S$ for $n, m \geqslant n_{0}$. In particular $x_{n_{0}}-x_{m} \in S$ for all $m \geqslant n_{0}$. Hence $x_{m}$ is in the finitely generated module $S+R x_{n_{0}}$ for all $m \geqslant n_{0}$. So the entire Cauchy sequence $\left(x_{n}\right)$ is in the finitely generated submodule $\tilde{S}=S+R x_{n_{0}}+\cdots+R x_{2}+R x_{1}$. We know that this finitely generated module $\tilde{S}$ is complete with the m-adic topology, and so by the above it is complete with the induced subspace topology it inherits from $M$. Since $\widetilde{S}$ is complete the sequence converges. Hence $M$ is complete. 


\section{REFERENCES}

[1] M.F. Atiyah and I.G. Macdonald, Introduction to commutative algebra (Addison-Wesley, Reading, MA, 1969).

[2] Belshoff, 'Matlis reflexive modules', Comm. Algebra 19 (1991), 1099-1118.

[3] N. Bourbaki, Commutative algebra (Addison-Wesley, Reading, MA, 1972).

[4] C. Chevalley, 'On the theory of local rings', Ann. Math. 44 (1943), 690-708.

[5] E. Enochs, 'Flat covers and flat cotorsion modules', Proc. Amer. Math. Soc. 92 (1984), 179-184..

[6] E. Matlis, 'Injective modules over noetherian rings', Pacific J. Math. 8 (1958), 511-528..

[7] H. Matsumura, Commutative ring theory (Cambridge University Press, Cambridge, 1986).

[8] M. Nagata, Local rings (Interscience, New York, 1962).

Department of Mathematics

Southwest Missouri State University

Springfield MO 64804-0094

United States of America 